SANDIA REPORT

SAND97-0072 • UC-705

Unlimited Release

Printed January 1997

\title{
LDRD Final Report on Enhanced Edge Detection Techniques for Manufacturing Quality Control and Materials Characterization
}

G. C. Osbourn

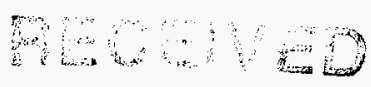

FFR 141997

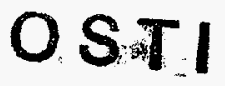

Prepared by

Sandia National Laboratories

Albuquerque, New Mexico 87185 and Livermore, California 94550

for the United States Department of Energy

under Contract DE-AC04-94AL85000

Approved for public release, distribution is unlimited.

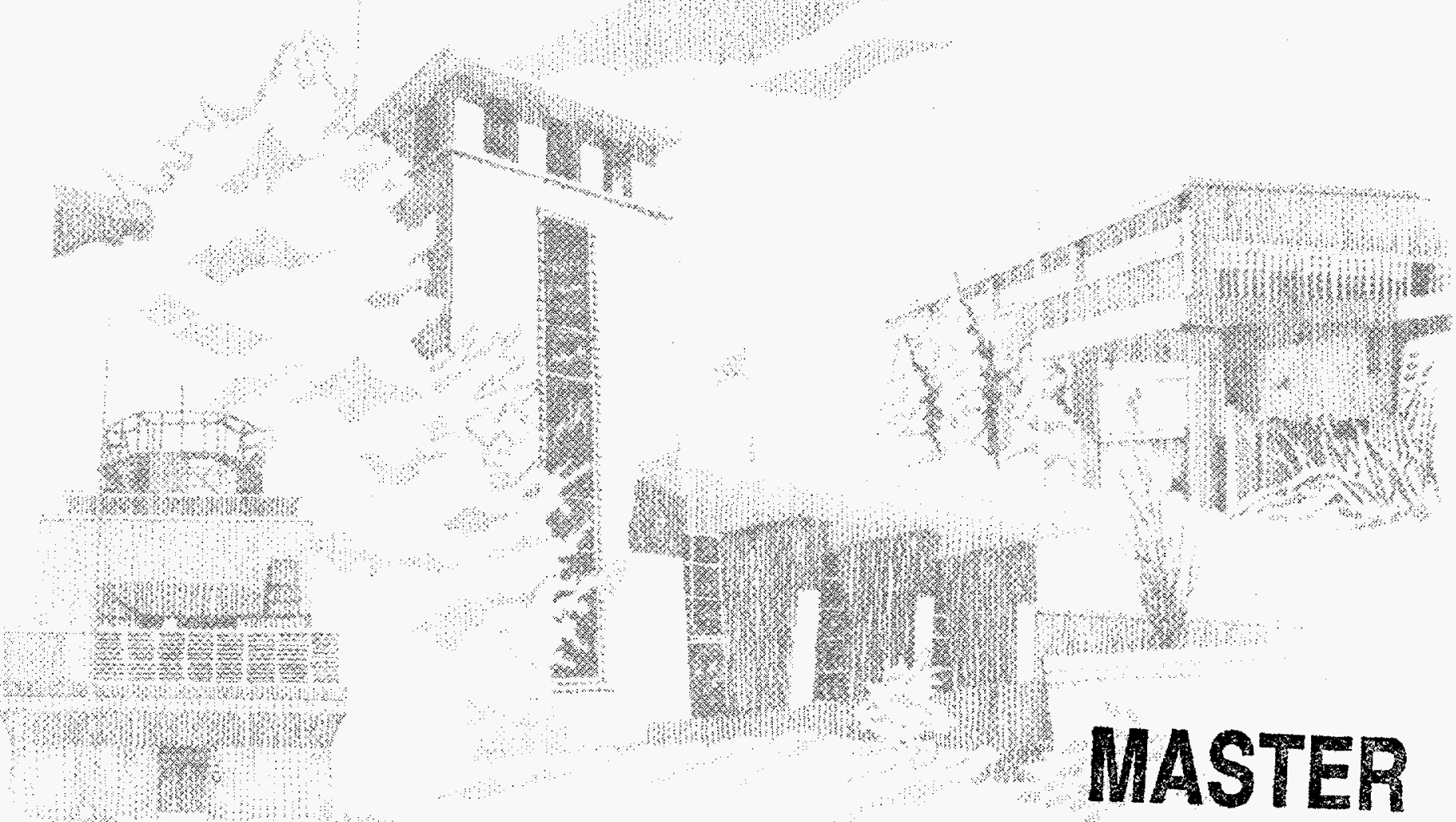


Issued by Sandia National Laboratories, operated for the United States Department of Energy by Sandia Corporation.

NOTICE: This report was prepared as an account of work sponsored by an agency of the United States Government. Neither the United States Government nor any agency thereof, nor any of their employees, nor any of their contractors, subcontractors, or their employees, makes any warranty, express or implied, or assumes any legal liability or responsibility for the accuracy, completeness, or usefulness of any information, apparatus, product, or process disclosed, or represents that its use would not infringe privately owned rights. Reference herein to any specific commercial product, process, or service by trade name, trademark, manufacturer, or otherwise, does not necessarily constitute or imply its endorsement, recommendation, or favoring by the United States Government, any agency thereof or any of their contractors or subcontractors. The views and opinions expressed herein do not necessarily state or reflect those of the United States Government, any agency thereof or any of their contractors.

Printed in the United States of America. This report has been reproduced directly from the best available copy.

Available to DOE and DOE contractors from

Office of Scientific and Technical Information

PO Box 62

Oak Ridge, TN 37831

Prices available from (615) 576-8401, FTS 626-8401

Available to the public from

National Technical Information Service

US Department of Commerce

5285 Port Royal Rd

Springfield, VA 22161

NTIS price codes

Printed copy: A03

Microfiche copy: A01 


\section{DISCLAIMER}

Portions of this document may be illegible in electronic image products. Images are produced from the best available original document. 
SAND97-0072

Unlimited Release

Printed January 1997

Distribution
Category UC-705

\title{
LDRD Final Report on \\ Enhanced Edge Detection Techniques for Manufacturing Quality Control and Materials Characterization
}

G. C. Osbourn, Vision Science, Pattern Recognition \& Multisensor Algorithms Dept.

Sandia National Laboratories

Albuquerque, NM 87185-1423

\begin{abstract}
Detecting object boundaries in the presence of cast shadows is a difficult task for machine vision systems. A new edge detector is presented which responds to shadow penumbras and abrupt object edges with distinguishable signals. The detector requires the use of spatially extended light sources and sufficient video resolution to resolve the shadow penumbras of interest. Detection of high frequency noise is suppressed without requiring image-dependent adjustment of signal thresholds. The ability of the edge operator to distinguish shadow penumbras from abrupt object boundaries while suppressing responses to high frequency noise and texture is illustrated with idealized shadow and object edge intensity profiles. Selective detection of object boundaries in a video scene with a cast shadow has also been demonstrated with this operator.
\end{abstract}


The goal of this project was to carry out basic exploratory research to improve the performance of certain machine vision algorithms. Machine vision offers the promise of significantly improving the quality and efficiency of the manufacturing process in a wide variety of industries. Effective vision systems can provide faster and more reliable product inspection, part identification and locating, measurements for process control, product sorting/grading, and detection of hazardous product defects which occur infrequently. Unfortunately, applications of vision technology in industry are hampered by poor performance and high costs of existing technology. The cost is partly a consequence of the inability of existing systems to handle general scenes with arbitrary lighting and shading. This forces expensive custom design of specialized systems with specialized lighting for nearly every company that uses the technology. The development of a more generally applicable vision approach could significantly reduce the cost of utilizing the technology by reducing the need for complete system re-design for every user.

Machine vision also could have a greater impact on advanced materials characterization techniques if improvements are achieved. A large number of advanced characterization techniques (e.g., SEM, TEM, photoluminescence imaging, infrared imaging, optical microscopy, EBIC, x-ray diffraction, RHEED, STM, etc.) provide important information on material structure and quality via two-dimensional images. Crystal growers, process developers and other material scientists often need fast, reliable repetitive and extensive quantitative results from these imaging techniques --- requirements that are difficult to meet through visual inspection of the images. Some of these images exhibit combinations of fine-detail features which the detection technique should find (e.g. dark line defects) and high frequency features which should be ignored (e.g. texture and noise). These properties are difficult to handle with existing edge detection techniques. The development of a more generally applicable vision approach would help automate these characterization techniques.

Machine vision also plays an important role in a variety of safeguards and military applications. Intrusion detection requires the rapid detection, position determination and evaluation of objects in the visual field. This detection process must be insensitive to changes in outdoor illumination and ideally should not be confused by shadows. Fast boundary detection is accomplished in some Sandia systems through motion detection, rather than by static edge detectors. However, the ability to separate the effects of illumination from object boundaries could provide improved performance by providing better object boundaries and object position for evaluating the type of intruder. This advantage would only be useful if an inexpensive, real-time implementation could be achieved.

This LDRD project focused on improving the low-level feature extraction operations of the machine vision algorithms, i.e. edge detectors, in order to develop new versions of these operators for detecting object boundaries and new operators for detecting fine-detail defects and features. The LDRD research focused on two key limitations of existing vision techniques which affect performance in these applications: sensitivity of existing edge detectors and template matchers to varying illumination conditions and shadows; inadequate selectivity of edge and template operators to specific spatial features, so that high contrast features with the wrong spatial properties can be confused with lower contrast features of interest. The most novel feature of this research was the use of knowledge about the human visual system to guide the development of the mathematical algorithms. In particular, our research attempted to determine what the effective "primitives" of the visual system were being computed from images in order to achieve the perception of object boundaries, shadow boundaries, and high spatial frequency details.

The primary success of this project was the development of two new edge detector operators which separately detect "isolated" object boundaries and "illumination" boundaries. An important aspect of these operators is that they depend on specific sets of spatial features for 
detection, rather than the strength of a derivative as in common edge detectors, and so are not fooled by other (non-edge) features that have high contrast. Separate detection of these features provided a considerable degree of immunity of the computed object edge results to varying illumination conditions, as observed in a variety of video images of scenes that we created with fixed arrangements of tools but varying light source positioning. This approach prevents shadow boundaries in the image from appearing as false object boundaries. The mathematical details of these operators are described in the appendix.

Some important capabilities remain to be developed in future work. Typical imaging conditions, such as the use of video cameras (which introduce noise) and the presence of very dark shadows, can prevent the operators from producing complete boundaries. Instead, the operators produce fragmented boundaries which contain gaps. It will be necessary to develop appropriate grouping algorithms to produce complete object and shadow boundaries from the results of these operators. The ability to mimic human perception of fine-detail, unlike the perception of object boundaries and illumination boundaries, remained incompletely understood at the end of this project. A key difficulty is the strong contribution of camera noise in the high spatial frequency range in which fine detail is detected. Approaches which worked well in distinguishing fine detail in simple imagery were observed to fail in the presence of video camera noise. It is the author's view that a better understanding of the human perceptual mechanisms associated with fine-detail perception will provide the most promising path for addressing this problem.

\section{Appendix}

\section{DETECTION OF OBJECT EDGES IN IMAGES CONTAINING CAST SHADOW}

\section{Introduction}

Edge detection and contrast perception have been extensively studied both from the machine vision (Roberts (1965), Prewitt (1970), Marr (1980), Rosenfeld (1970), Nalwa (1986), Torre (1986), Haralick (1984), Canny (1986)) and the psychophysical (Campbell (1974), King-Smith (1975), Hall (1977)) points of view. Few of these studies have addressed the problem of distinguishing illumination variations (e.g. shadows) from reflectance variations. This problem is quite difficult since the eye and the video camera receive only the combined effects of illumination and reflectance. For example, it is not clear whether a dark region in an image is due to the presence of a shadow (weak illumination) or a dark object (poor reflectance). Nevertheless, the human visual system is remarkably successful at separating these effects and estimating the reflectance magnitudes of points in a scene (Gilchrist (1984), Rock (1984)). Machine vision systems are typically not robust against varying illumination conditions such as are encountered in normal outdoor scenes. The attention to lighting methods which is required in industrial vision applications (Batchelor (1985)) further attests to the sensitivity of machine vision systems to illumination conditions. A method for separating the variable effects of changing illumination conditions from the typically invariant reflectance

properties of physical objects should add desirable robustness to machine vision systems. In this appendix we address the specific case of detecting abrupt object boundaries in video scenes which also contain cast shadows.

One possible cue for recognizing cast shadow boundaries is the presence of spatially extended penumbras at the borders of shadows. Most light sources which are encountered (e.g. the sun, moon, incandescent and florescent bulbs, flames) are in fact physically extended, and these extended light sources create shadow boundaries that are not abrupt. Any illuminated object completely blocks the direct illumination from the source in the main region of the shadow and blocks none of the direct illumination at locations sufficiently far away from the shadow, but the 
shadow penumbra between these two regions is only partially blocked from the illumination source. The intensity in the penumbra linearly increases (for a uniform source) as less of the extended light source is blocked, and the width of the penumbra region depends on the angular extent of the light source and the separation of the opaque object and the surface that the cast shadow falls on. This intensity profile is schematically illustrated in Fig. 1.

The ramp profiles of shadow penumbras are unlike the boundaries associated with most physical objects. Such physical boundaries are often quite abrupt, and give rise to intensity profiles such as that illustrated in Fig. 2. These physical boundaries typically give rise to reflectance differences between the objects and their backgrounds (which do not depend on illumination conditions) and, for three dimensional objects, can also give rise to illumination differences across the boundary. The profile in Fig. 2 might represent the slightly shaded edge of an object, where the degree of contrast difference across the boundary due to highlights or shading on either side depends on the lighting conditions but the presence of the boundary occurs independently of lighting conditions due to the reflectance difference.

The differing spatial configurations of the ramp-like shadow boundaries and the abrupt object/reflectance boundaries offer a means for distinguishing shadow boundaries and abrupt object/reflectance boundaries and further suggest that edge detection techniques might be useful for this purpose. In fact, the human visual system produces distinguishable perceptual responses at these two types of intensity boundaries. The abrupt object boundaries (e.g. Fig. 2) are correctly perceived as abrupt intensity edges, while the shadow penumbras are incorrectly perceived as containing additional illusory bright and dark Mach bands (Ratliff (1965)). The Mach bands can be seen in the gray scale version of Fig. 1 (not shown) and their apparent positions on the intensity plot are labeled by arrows. The intensity values perceived in these bands are not real. Mach bands have long been regarded as artifacts associated with lateral inhibition in biological visual systems (Ratliff (1965)). Lateral inhibition is a form of second derivative operation which acts on the input intensities (Levine (1985)). Fig. 1 illustrates that the positions of the Mach bands occur roughly where the second derivative of the intensity profile is nonzero.

Here we present a new edge operator which is designed to respond at the boundaries of shadow penumbras and at abrupt object boundaries with distinguishable signals at each type of boundary. The penumbra boundary signals are patterned after the human perception of Mach bands and label the expected presence of illumination changes. Signals at the abrupt edges are analogous to the human perception of object boundaries and label the expected presence of reflectance changes. Insensitivity to high frequency noise and texture is obtained in a way which avoids the use of low-pass blurring filters, so that the distinction between abrupt and spatially extended edges is not filtered out.

\section{Edge Operator}

The edge detector is defined as follows. Directional second difference values defined by

$$
D^{2} I(x)=I Æ(x)-2 I Æ(x+Æ)+I Æ(x+2 Æ) \quad(E q .1)
$$

at each pixel location $\mathrm{x}$ in a row (on a grid of pixel width $Æ_{0}$ ) are examined for the following conditions:

$$
\left|\mathrm{D}^{2} \mathrm{I}_{\circledast}(\mathrm{x}-Æ)\right|>\left|\mathrm{D}^{2} \mathrm{I} Æ(\mathrm{x}-Æ / 2)\right|>\left|\mathrm{D}^{2} \mathrm{I} Æ(\mathrm{x})\right|
$$

and

$$
\operatorname{sign}\left(D^{2} I_{Æ}(x-E)\right)=\operatorname{sign}\left(D^{2} I_{Æ}(x-Æ / 2)\right)
$$


The $I_{Æ}(\mathrm{x})$ values are intensity averages over a width $Æ$ along a row of pixels. The second difference operators model the effects of the receptive fields which produce lateral inhibition in the human visual system (Levine (1985)), and each trio of adjacent pixels corresponds to one receptive field. The conditions of Eq. 2 are satisfied at image locations where the second difference values are approaching zero. The requirements on the signs of the two $\mathrm{D}^{2} \mathrm{I}$ also serve to reduce the aliasing response of the operator to some high frequency components. These conditions on the second difference operators are examined for a set of effective pixel width values. The use of multiple $\mathbb{E}$ values increases the spatial frequency bandwidth of the edge detector response. Large $Æ$ values are obtained for the video operator by averaging intensities over a set of adjacent pixels. The pixel widths are

$$
\nexists=\mathrm{m}^{*} \mathbb{E}_{0}, \mathrm{~m}=2, \ldots, 8 \quad \text { (Eq. 3) }
$$

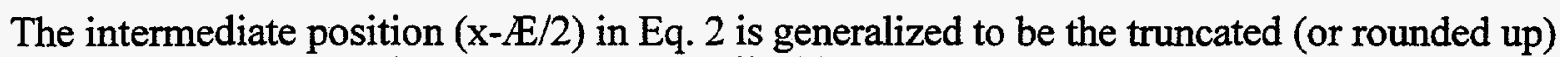
integer value for the $+/ 0$ (or $0 /+$ ) case described below when the pixel width $Æ$ is an odd integer in the video case.

If the above conditions are satisfied for a particular $Æ$, then the total edge signal (Signal( $\mathrm{x})$ ) is increased by the contribution:

$$
\begin{aligned}
& \operatorname{Signal} Æ(x)=\operatorname{sign}\left(D^{2} I Æ(x-E)\right)^{*} \max \left\{\left|D I_{Æ}(x-E)\right|,\right. \\
& |\mathrm{DI} \nsubseteq(\mathrm{x})|,|\mathrm{DI} Æ(\mathrm{x}+Æ)| \xi /\left(\mathrm{I}_{\mathrm{m} / 2)} \quad\right. \text { (Eq. 4) }
\end{aligned}
$$

where

$$
\mathrm{DI} Æ(\mathrm{x})=\mathrm{I}_{\circledast}(\mathrm{x}+Æ)-\mathrm{I}_{Æ}(\mathrm{x}) .(\mathrm{Eq} .5)
$$

$\mathrm{I}_{\mathrm{m}}$ is the difference between the largest and smallest intensity values in the entire image. The total Signal(x) is the sum of the $\operatorname{Signal} Æ(x)$ for the set of $Æ$ values, provided that the $\operatorname{Signal} Æ(x)$ contribution from the operator with the smallest effective pixel width $\left(=2 \Xi_{0}\right)$ is nonzero, otherwise the Signal total at position $\mathrm{x}$ is set to zero. This condition is necessary to prevent multiple replicas of a single edge due to the use of multiple $Æ$ values. Two separate Signal(x) sums are maintained, with the second sum corresponding to using $\mathbb{E}$ 's in Eq. 3 with negative signs. These two sets of signals will be represented as $+/ 0$ and $0 /+$, and correspond to requiring that the second difference magnitudes decrease to the right ( $+/ 0$, positive $Æ$ values) or to the left $(0 /+$, negative $E$ values). The $+/ 0$ and $0 /+$ signals are separately examined for edges along the pixel rows, and one edge is assigned at the location of the largest absolute value in each set of adjacent nonzero signal pixels with the same sign.

The issue of extending these one-dimensional equations to two dimensions is handled by making row and column one-dimensional scans through the video image. Conflicts between edges detected vertically and those detected horizontally are handled by accepting abrupt edges over Mach band edges and accepting the edge with the largest signal magnitude if both are abrupt or Mach band types with the same sign. In principle it is possible to include various diagonal scans, but that possibility is not considered further here. 


\section{Spatial properties of computed edges}

Fig. 3 illustrates the types of edge signatures that are produced at penumbra boundaries and at abrupt contrast edges. The idealized intensity profile is shown at the top of the figure and the corresponding computed $+/ 0$ and $0 /+\operatorname{Signal}(\mathrm{x})$ values are shown at the bottom. Arrows indicate the positions of computed edges occurring at the peaks of the Signal responses. We note that a slightly modified set of pixel widths (which are intended to better model the human perception of Mach bands) rather than that in Eq. 3 was actually used for the results in this figure and Figs. 4-5. The penumbra ramp at left produces two closely spaced pairs of edges, one straddling each end of the ramp. Each pair consists of a $+/ 0$ edge and a $0 /+$ edge, both with the same sign. These pairs of peaks occur roughly at the boundaries of where the illusory Mach bands are perceived, with negative (positive) signal edges corresponding to bright (dark) bands, respectively.

The abrupt contrast edge at right produces a coincident pair of $+/ 0$ and $0 /+$ edge peaks with opposite signs. Since the two signal peaks occur at the same location, only one edge is produced. Note that the abrupt contrast edge can be thought of as the limiting case of the penumbra ramp as the ramp width decreases to zero. One might expect that bringing together the edges at the left of the figure would produce two coincident edges with opposite signs (which is obtained at right) plus two more edges on either side of the first pair. In fact, all previous quantitative models of Mach bands predict exactly that result (Ratliff (1984)). However, humans typically do not perceive Mach bands at abrupt edges. This discrepancy has been a long standing puzzle in the field of Mach band study (Ratliff (1984)). The present model suppresses the outer edges through the high frequency aliasing suppression action inherent in Eq. 2. As the penumbra ramp width goes to zero, the inner edges increase in Signal magnitude while the outer edges decrease, until the inner edges merge and the outer edges vanish. Thus, the present model produces no calculated Mach bands at abrupt contrast edges, in agreement with human perception. This result simultaneously resolves the long standing theoretical difficulty with lateral inhibition action on abrupt edges and provides a signature for distinguishing computed Mach edges from computed abrupt contrast edges. This latter result follows from the qualitative differences between nearby Mach bands (with nonoverlapping $/-$ and $+/+$ edge peaks) and abrupt contrast edges (with overlapping $-/+$ edge peaks). Note that these $+/$ - edge peaks are related to the zero crossings of the second derivative which form the basis of many prior edge detector methods (Marr (1980), Torre (1986), Haralick (1984)).

Figs. 4 and 5 explicitly demonstrate that suppression of signals from some high spatial frequency noise and texture is intrinsic to the edge operator. The idealized intensity profile at the top of Fig. 4 shows an abrupt contrast edge and a shadow penumbra, both of which are corrupted with high frequency noise signals. The computed edge signals at the bottom of the figure look qualitatively similar to those obtained in Fig. 3. No edge signals are produced by the high frequency signals due to the conditions in Eq. 2. Fig. 5 contains a set of abrupt edges with varying proximity to each other. The closest pair corresponds to a single pixel with a low intensity, and this pair produces strongly suppressed edge signals due to the aliasing suppression effects of Eq. 2 . As the distance between neighboring abrupt intensity changes is increased, first one edge signal turns on and then both of the $+/$ - pair signals are nonzero. These results illustrate that the operator responds strongly to relatively isolated abrupt edges but responds weakly or not at all to higher frequency edge signals.

\section{Noise thresholds for the edge operator}

Figs. 4 and 5 illustrate that some high frequency signals are ignored by the edge operator. However, to obtain a more complete picture of the high frequency noise response it is necessary to examine the frequency-dependent response of the operator to idealized penumbra waveforms (trapezoids) and abrupt edge (sawtooth; square wave) waveforms as described in Osbourn (1991). Fig. 6 shows the strengths of the calculated edge signals from these three waveforms at 
unity contrast as a function of spatial frequency. The results show a desirable signal response at the lower frequencies, a gap in the sensitivity where no edges are detected, and some residual edge detection at high frequencies due to undesirable aliasing. The aliasing signals at frequencies greater than $0.75 / £$ have waveform periods less than the smallest pixel width of the operator. The aliasing response can be suppressed in an optimal way (i.e., with minimum reduction of the observed low frequency signal strength) by selecting a Mach edge threshold at the signal magnitude given by the horizontal dashed line in the figure. This threshold is at the maximum signal strength of the aliasing response of intensity patterns with the strongest edges (those with abrupt contrast edges). Thus signal strengths above this threshold in the figure must be due to low frequency patterns. Note that this threshold is not an adjustable parameter, but is determined entirely from the computed aliasing response to intensity patterns with the strongest aliasing signals. The sensitivity to shadow contrasts (as represented by trapezoidal penumbra intensity patterns) obtained in this way compare favorably with measured human vision values. However, the sensitivity to abrupt edges obtained in this way is nearly a factor of 10 smaller than those of the human visual system.

A modest improvement in the sensitivity can be obtained by noting that the low frequency responses to intensity patterns with abrupt contrast edges reach a plateau with a large signal magnitude which continues indefinitely with decreasing frequency. This unique low frequency response occurs because all of the individual operators with different $\nexists$ values respond strongly to relatively isolated abrupt edges. Fig. 7 illustrates a quantity which represents the effective number of responding operators (ENRO), defined by

$$
\mathrm{ENRO}=\left(\operatorname{Signal}_{\mathrm{E}}\right) / \max \left\{\text { Signal }_{\mathrm{E}}\right\}
$$

where the summation and the maximum are over all $Æ$ values. ENRO values for square and sawtooth patterns are shown in the figure. This parameter can have a maximum value of seven, equal to the number of pixel sizes used in the model. Note that this value is achieved when all operators respond with equal strength, not when all operators respond with arbitrary signal levels. ENRO values are measures of the frequency bandwidths associated with edges, so that abrupt edges with many large-amplitude Fourier components produce the strongest ENRO values. The ENRO values are also qualitatively similar to the signal strengths for the case of abrupt intensity edges. The ENRO values in the aliasing frequency regime reach values of 7 at $\mathrm{f}=1 / \AA_{0}, 2 / Æ_{0}$, etc. This is a consequence of using integer pixel sizes, and could in principle be avoided if a variety of noninteger pixel widths were available. The alias signals from the operators with integral pixel widths coherently add up at multiples of the frequency $\left(1 / \mathbb{E}_{0}\right)=1$. Examination of the Mach signal strengths at the frequencies where the alias ENRO values are large reveals that the signals are generally small and nearing zero. This suggests a useful tradeoff approach for enhancing the abrupt edge sensitivity while minimizing the amount of aliasing signal detected. In particular, if a reasonably large ENRO threshold is artificially selected, then only the alias frequencies with larger ENRO responses and modest signal values need further attention. The response from these alias frequencies can be further suppressed by introducing an additional signal threshold for the edge peaks which are identified as abrupt edge signals. Values of 4.8 and 0.2 are used for the ENRO and abrupt edge signal thresholds, respectively, in this work. The abrupt edge signal threshold is lower than the 0.6 value required for Mach edges, so that the abrupt edge detection sensitivity is somewhat improved with this modified video ENRO/signal threshold approach. However, the improvement still falls short of that achieved by human vision. Other methods for further improving the sensitivity of the abrupt edge detector will be the subject of future work. 


\section{Video results}

The performance of the video version of this edge detection approach has been examined by computing object and shadow boundaries for laboratory images of simple objects and tools (a pencil, an Allen wrench, a file). The lighting source is varied in location to create different shadow patterns for fixed object locations. The computed results demonstrate that the cast shadows are successfully ignored by the object boundary edge operator. The abrupt edges of the objects are successfully detected except where the shadow occludes them or where the edges are not well isolated (as in Fig. 5). As discussed in Osbourn (1991), additional operators are needed to selectively detect high frequency texture and closely spaced edges. It should be noted that the Allen wrench and the file as well as parts of the pencil are as dark as the interior of the cast shadows. This illustrates that darkness alone cannot in general be used to successfully recognize the presence of shadows in real video images.

\section{Conclusions}

An edge detection approach for selectively detecting abrupt contrast edges in the presence of cast shadows is presented. The edge detector produces qualitatively distinguishable edge signatures at the linear ramp intensity boundaries of shadow penumbras and the sharp intensity jumps of abrupt object/reflectance boundaries. A key element of this approach is a method for suppressing noise which does not require low-pass filtering of the image prior to edge detection, so that abrupt edges are not blurred before edge detection takes place. The high frequency noise suppression properties of the operator have been explicitly demonstrated from both the real-space and frequency-space points of view. The edge operator is applied to a representative video image with multiple objects and a cast shadow. The video results verify that abrupt object/reflectance boundaries are detected and shadows are successfully ignored while maintaining insensitivity to high frequency noise.

\section{References}

Batchelor, B. G., Hill, D. and Hodgson, D. C. (editors) (1985). Automated Visual Inspection, New York, Elsevier Science Publ.

Campbell, F. W. and Maffei, L.(1974). "Contrast and Spatial Frequency", Scientific American 231 , no. 5, pp. 106-114, Nov.

Canny, J. (1986). "A computational approach to edge detection" IEEE Trans. Patt. Analysis and Mach. Intell. PAMI-8, no. 6, pp. 679-698.

Gilchrist, A. and Jacobsen, A. (1984). "Perception of lightness and illumination in a world of one reflectance", Perception 13, pp.5-19.

Hall, C. F. and Hall, E. L. (1977). "A nonlinear model for the spatial characteristics of the human visual system", IEEE Trans. on Sys., Man and Cyber., SMC-7, no. 3, pp.161-170.

Haralick, R. M. (1984). "Digital step edges from zero crossings of second directional derivatives" IEEE Trans. Patt. Analysis and Mach. Intell. PAMI-6, no. 1, pp. 58-68.

King-Smith, P. E. and Kulikowski, J. J. (1975). "The detection of gratings by independent activation of line detectors", J. Physiol. 247, pp. 237-271.

Levine, M. D. (1985). Vision in Man and Machine, New York, McGraw-Hill. 
Marr, D. and Hildreth, E. (1980). "Theory of edge detection", Proc. Royal Soc. London, ser. B, 207, pp. 187-217.

Nalwa, V. S. and Binford, T. O. (1986). "On detecting edges", IEEE Trans. Patt. Analysis and Mach. Intell. PAMI-8, no. 6, pp. 699-714.

Osbourn, G. C. (1991). "Distinguishing cast shadow boundaries from abrupt object boundaries via edge detection", Proc. SPIE 1614, pp. 63-77.

Prewitt, J. M. S. (1970). "Object enhancement and extraction" in Picture Processing and Psychopictorics, Lipkin, B. S. and Rosenfeld, A., (editors), New York, Academic Press.

Ratliff, F. (1965). Mach bands: quantitative studies on neural networks in the retina, San Francisco, Holden-Day.

Ratliff, F. (1984). "Why Mach bands are not seen at the edges of a step", Vis. Res. 24, no. 2, pp 163-165.

Roberts L. G. (1965). "Machine perception of three-dimensional solids" in Optical and Electrooptical Information Processing, Tippett, J. P. et. al. (editors), Cambridge MA, MIT Press.

Rock, I. (1984). Perception, New York, Scientific American Books.

Rosenfeld, A. and Thurston, M. (1970). "Edge and curve detection for visual scene analysis", IEEE Trans. on Comp. C-20, no. 5, pp. 562-569, May.

Torre, V. and Poggio, T. A. (1986). "On edge detection", IEEE Trans. Patt Analysis and Mach. Intell. PAMI-8, no. 2, pp. 147-163.

IATENSITY PROFILE

AND

PERCEIYED EDGES

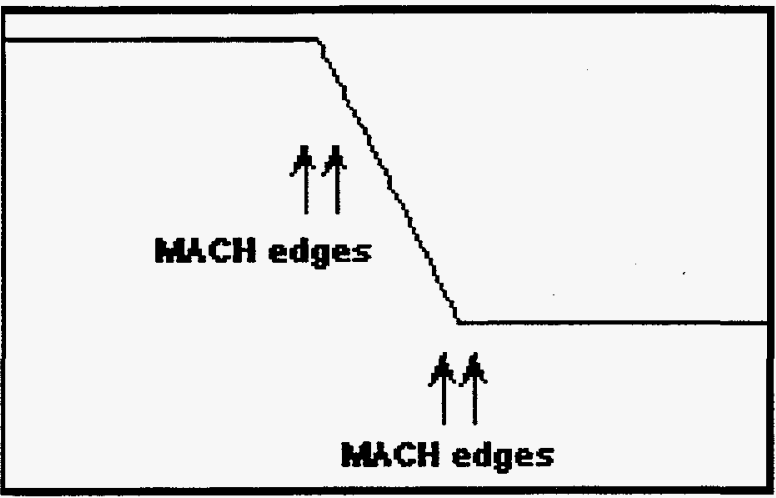

Fig. 1 Illusory Mach bands at the borders of an ideal (linear intensity ramp) shadow penumbra. The image at top shows the intensity variation in a shadow penumbra and the schematic diagram indicates the intensity magnitude as a function of lateral position. Illusory bright and dark Mach bands are indicated by arrows at the top, and the approximate positions of the pairs of Mach edges associated with these bands are indicated by arrows in the bottom diagram. Inspection of the true intensity pattern in the diagram at bottom verifies that the apparent intensities in the Mach bands are not real. 
IHTERSITY PROFILE

AHD

PERCEIYED EDGE

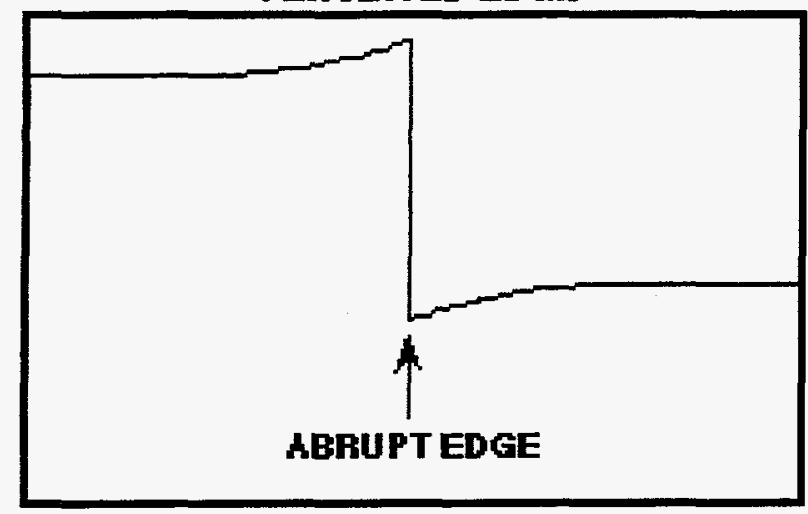

Fig. 2 Abrupt contrast edge indicated directly in the top image and schematically in the bottom diagram. Object and reflectance boundaries typically produce intensity patterns approximately like that shown in the figure.

MACH AND ABRUPT EDGES

DETECTED AND DISTINGUISHED

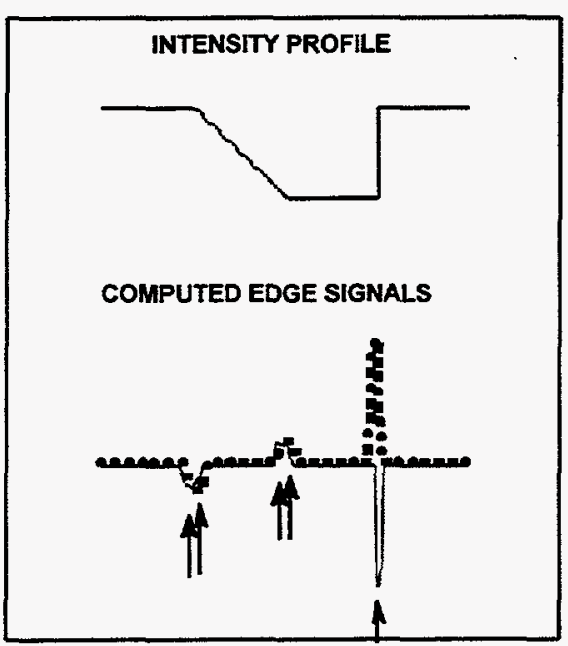

Fig. 3 Computed edge signals (bottom) for an intensity pattern (top) which includes a linear ramp and an abrupt contrast edge. The $+/ 0(0 /+)$ signals are given by the solid (dotted) lines, respectively. The peaks of the edge signals determine the positions of the computed edges as indicated by the arrows. The ramp at left produces two Mach bands (four Mach edges total) and the abrupt contrast edge produces two edge peaks at the same position (i.e. one discernible edge). 


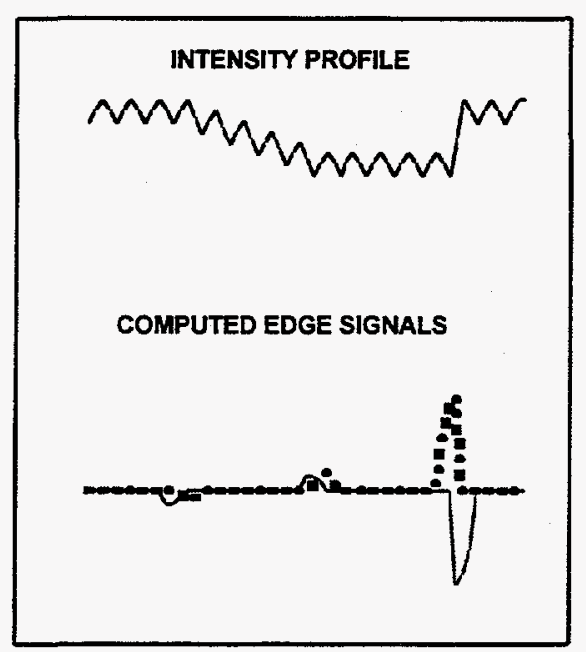

Fig. 4 lllustration of the insensitivity of the edge operator to high spatial frequency noise. The high frequency signal has a period of $2 \mathbb{E}_{0}$ and is superimposed on an intensity pattern like that of Fig. 3. The $+/ 0(0 /+)$ operator signals are represented by solid (dotted) lines, respectively, in the bottom portion of the figure. The closely spaced high frequency edges are ignored by the operator, but the Mach band edges and the abrupt contrast edge are detected.

\section{CLOSELY SPACED ABRUPT EDGES \\ SUPPRESS DETECTION}

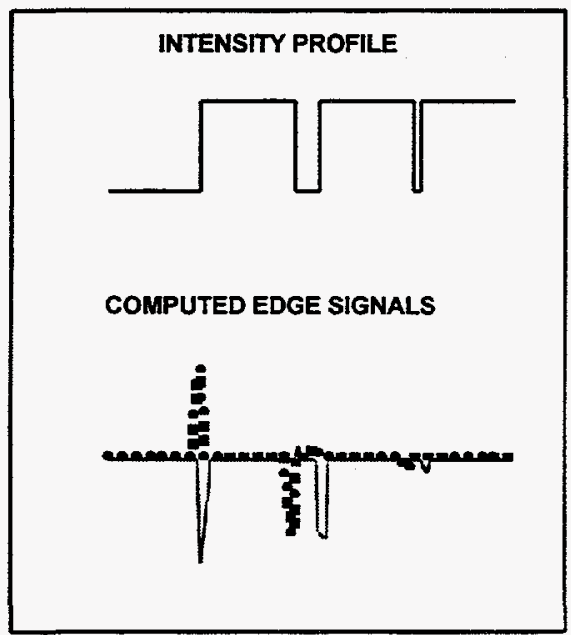

Fig. 5 Illustration of the insensitivity of the edge operator to closely-spaced abrupt contrast edges. This results from the alias suppression properties of the edge operator. The intensity profile is at the top of the figure and the computed edge signals are at the bottom. The $+/ 0(0 /+)$ signals are represented by solid (dashed) lines, respectively in the bottom portion of the figure. The relatively isolated abrupt contrast edge at left produces the usual pair of coincident computed edges. The pair of abrupt contrast edges in the middle are close enough to each other to inhibit the signal from one of the coincident edge peaks, leaving a single strong edge peak for each abrupt contrast edge. The pair of abrupt contrast edges at the right are associated with a singlepixel-wide dip in the intensity. These edges are so closely spaced that all of the edge peak signals are strongly suppressed. 
VIDEO MODEL

EDGE SIGNALS

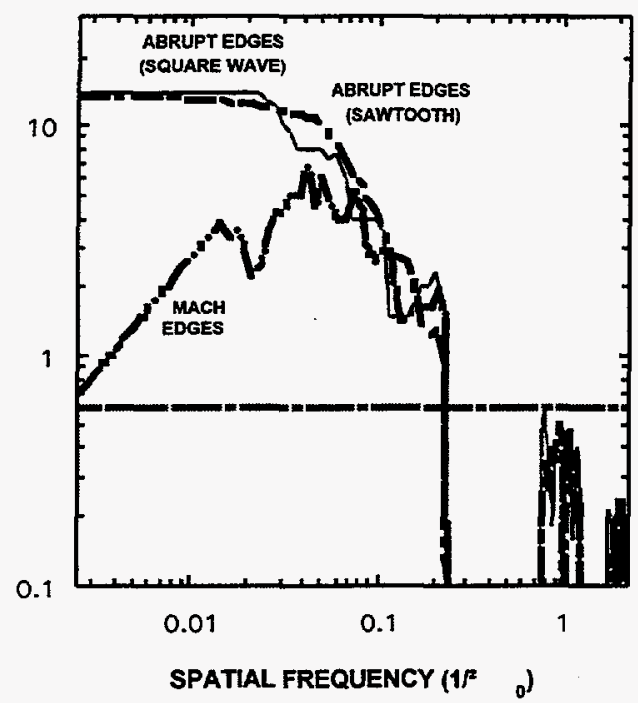

Fig. 6 Computed edge strengths from the edge operator. The low frequency signals are for a trapezoid pattern (Mach edges) and for the square wave and sawtooth wave patterns (abrupt contrast edges), and the alias signals at high frequencies are from the square wave and sawtooth patterns. The horizontal dashed line gives the Mach signal threshold.

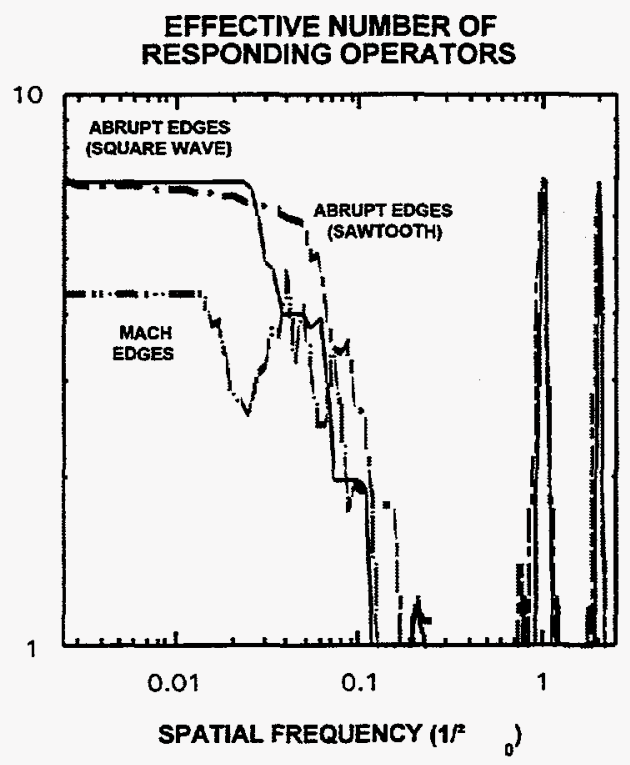

Fig. 7 ENRO (effective number of responding operators) for the edge operator. The limitation of integer pixel width values causes the alias responses from the different individual second difference operators to add coherently as the frequency approaches multiples of $\left(1 / \varpi_{0}\right)=1$. 
Distribution:

$\begin{array}{lll}20 & \overline{\text { MS }} & \text { Name, Org. } \\ 1 & 0188 & \text { G. C. Osbourn, 1155 } \\ 1 & 9018 & \text { C. E. Meyers, 4523 } \\ 5 & 0899 & \text { Technical Tech Files, 8940-2 } \\ 2 & 0619 & \begin{array}{l}\text { Review and Approval Deval Des, } 12690 \\ \text { for DOE/OSTI }\end{array}\end{array}$

